\title{
OGM et le droit de la consommation: une action mondial pour assurer l'information des consommateurs?
}

\author{
Claudia Lima Marques ${ }^{1}$
}

\section{Introduction}

Les avantages de l'évolution de la biotechnologie sont payés d'un lourd tribut de risques. De nowveaux progrès scientifiques, comme les aliments et les denrées alimentaires génétiquement modifiées, le "Novel Food", 2 propose un sérieux défi pour l'actuel droit de la consommation. On peut définir les organismes génétiquement modifiées (OGM) conme "un organisme, à l'exception des êtres humains, dont le matériel génétique a été modifié d'une manière qui ne s'effectue pas naturellement par multiplication et/ou par tecombinaison naturelle". 3

La question de savoir si les OGM ou une de leurs utilisations comme ingrédient des produits alimentaires ou comme aliment animal, peuvent ou non causer des effets néfastes aux consommateurs, maintenant ou dans le futur, ri'est pas encore scientifiquement

\footnotetext{
'Dr. iur. (Heidelberg), LL.M. (Tübingen); Professeur Titulaite de Droit Intemational Privé de l'Université fédéral Rio Grande do Sul, Porto Alegre, Brésil. L'auteur remercie vivement Ana Rispoli de Azevecto (FAPERGS/UFRGS) et Christian Schindler (Univ. Heidelberg) pour le matériel et les amies António Herman Benjamin (MP/SP) et Silvia Capelli (MP/RS) pour les idées et les fructueuses discussions.

${ }^{2}$ Voir Règlement CEE 258/97, de $15 \mathrm{Mal} 1997$, le "Novel Food"-Verordunung, in www. verbraucherministerium.de. Voir Règlement (CE) n, 258/97, JO L 043 du 14.02.1997, p. 0001, en français "nouveaux aliments", voir aussi BJERREGARD, Ritt, Labeling of Novel Food-Providing full information to consumers, in European Food Law Review, 1998, p. 1 ss.

${ }^{3}$ Définition de h'article 2,2 de la Directive 2001/18/CE, du 12 mars 2001, Journal Officiel des Communautés Européennes de 17.4.2001, L 106/4.
} 
sûre. ${ }^{4}$ On travaille avec des probabilités, des essais, des évaluation des risques, des tests, qui jusqu’à présent soulignent la nécessité de précaution. ${ }^{5}$ En effet, un souci de sécurité anime presque tous les pouvoirs publics et les institutions internationales lors l'elaboration de législations propres à protéger la santé publique, les consommateurs et l'environnement en cas de plantation des semences-OGM, de mise en circulation sur les marchés de consommation des OGM destinés à l'alimentation humaine (directement, comme les tomates Flavr-Savt, ou indirectement comme des ingrédients de produits alimentaires, le soja ou le maïs $O G M$ ) ou de la mise en circulation des produits destinés à lalimentation animale (ainsi les mä́s et soja OGM, qui posent le problème de la traçabilité de ses produits). La doctrine a développé la théorie de l'évaluation divergente des risques (Streinz), qui précomse précaution et information lorsque les évaluations des risques en l'état actuel de la science sont encore divergentes, comme c'est le cas pour les $O \mathrm{GM}^{6}$

Au début du XXème. siècle été développé le concept d'obligation de sécuritế pour protéger la faiblesse de l'homme vulnérable devant des forces techniques susceptibles de porter atteinte à son intégrité physique. ${ }^{9}$ Au cours des années 70 , la spécificité de la tesponsabilité du fait des choses et, notamment, du fait des produits industriels défectueux, a abouti à une série d'initiatives réglementaires concernant: a) la prévention des accidents de consommation; b) l'étiquetage et l'information aux consommateur; c) l'uniformisation des règles matérielles sur le responsabilité du fait des produits et d) la fléxibilité des règles de droit international privé spéciales aux dommages de consommation.

Voyons quelques unes de ces fructueuses initiatives.

En droit interne, le législateur a concentré ses efforts sur le combat contre les

\footnotetext{
"Voir sur l'étique de l'environnement', ROSE, Carol M., Scientific inovation and environnenental protection: some ethical consideration, in Environnemental Law, vol. 32, Fall, Number 4, p. 755 ss. Voir études nord-américains de Ia Food and Drug Administration et critiques, in http:// www biointegrity.org

S BENJAMIN, Antônio, Função Ambiental, in Revista de Direito Ambiental, vol. 2, 1993, p. 11.

"Voir sux le "Theorie der divergierenden Risikoabschätzung", développée par STREINZ, Rudolf, Divergierende risikoabschätzung und Kennzeichung, in STREINZ (Ed.), Novel Food, 2.Ed, Bayreuth, 1995, p. 131ss, l'étude de KNÖRR, Georg Oliver, Die Kennzeichnungspflicht gentechnisch veränderter Lebensmittel, Köhler-Druck, Tübingen, 2000, p. 90ss.

'Selon LAMBERT.FATVRE, Yvonne, Fondement et régime de l'obligation de sécurité, Recueil Dalloz, 1994, p. 81.

"Voir sur le rôle de la jurisprudence française dans le développement de cette notion, MELLER, Eberhard, Obligation de Sécurité - Verbesserung des Schutzes des geschädigten Vertragspartner und Beispiel der Fortentwicklung des französischen Vertragssechts durch die Rechstprechung, Duncker \& Humblot, Berlin, 1973, p. 16ss.

- Voir BOULOC, Bernard, La loi de 1905 en tant qu'instrument de la sécurité des consommateurs, in GHESTIN, Jacques (Org.), Sécurité des consommateurs et responsabilité du fait des produits défectueux,LGDJ, Paris, 1987, p. 13 ss.
} 
falsifications, ${ }^{16}$ sur la prévention des fraudes et sur l'élaboration d'un cadre législatif pour l'information des consommateurs. Le principal exemple en est la loi française. Loi 78-23 de janvier 1978 sur la protection et l'information des consommateurs sur les produits et les services, on citera également la prévention des fraudes par l'étiquetage correct des produits. "

Dans les institutions internationales, la première initiative fut celle de la Conférence de La Haye de Droit International Privé, qui a organisé la 'Convention sur la loi applicable à la tesponsabilité du fait des produits' du 2 octobre $1973 .{ }^{12}$ Dans le cadre du Conseil de l'Europe, les pays membres ont organisé la Convention du 27 janvier 1977 sur la responsabilité du fait des produits en cas de lésions corporelles ou décès, visant la protection des victimes de produst défectueux par I'unification des règles matérielles de responsabilité ${ }^{13 i}$ la Convention de Strasbourg de 1977 a introduit la notion de défaut et montre une tendance vers une responsabilité plus stricte des producteurs en Europe, raison pour laquelle la Convention de Strasbourg est considérée comme source historique et technique de la Directive européenne de $1985 .^{14}$

A l'OCDE ont été élaborées la Recommandation du Conseil concernant la sécurité des produits de consommation du 18 décembre $1979^{15}$ et la Recommandation du Conseil concernant l'établissement de systèmes de recensement des données relatives aux accidents provoqués par des produits de consommation du 13 février $1978,{ }^{16}$ et pour l'étiquetage, les directives concernant les contrôles de sécurité sur les cosmétiques et sur les produits ménagers. ${ }^{17}$ La Communauté Européenne a travaillé la première version de la fameuse Directive sur la

\footnotetext{
BOUlOC, p. 15

"Voir édition du journal Officiel de la République française. Répression des fraudes et sécurité des consommateurs - Textes législatifs et réglementaires. Edition novembre 1991, Paris, 1991, p. 4ss. Avec la Loi $78-1978$ a la p. 13-17.

12 WANDT, Manfried, Internationale Produkthafturig, Verlag Recht und Wirtschaft, Heidelberg. 1995 , p. $58 \mathrm{ss}$. La convention a été ratifiée par la France, la Norvège, lancienne Yougoslavie, les Pays Bas, le Luxembourg, I'Espagne et la Finlande. WANDT, p. 58.

13 MARKOWITZ, p. 53ss.

14 Voir aussi MARKOWITZ, p. 66-67

15 OCDE, La politique à l'égard des consommateurs au cours des dix dernières années - Evolution et pespectives, Bd. OCDE, Paris, 1983, p. 89-91.

16 OCDE, op.cit, p. 77-81.

17 OCDE, op.cit. p. 60667 .
} 
responsabilité du fait des produits défectueux dès $1976,{ }^{18}$ et a imposé une responsabilité sans faute, dans un premier temps avec l'exclusion explicite des produits naturels du champ d'application de la Directive de $1985 .{ }^{12}$

Il a semblé, pendent un certain temps, que l'influence du droit nord-américain et sa strict product liability ${ }^{20}$ ainsi que celle du droit harmonisé européen avec sa notion de défaut, ${ }^{21}$ reprise par le Code brésilien de Défense du Consommateur (Loi 8.078/90 Art. 1214), ${ }^{22}$ pouvait être suffisante pour assurer sur le marché mondial l'écoulement des produits non dangereux. La crise de 'vache folle' en Europe a montré que la prévention des dommages de consommation est plus importante que des règles strictes de responsabilité. La fonction préventive d'une responsabilité aggravée existe, mais elle est d'une efficacité discutable. ${ }^{23}$ Les risques dans la societé acruelle se sont transformés en risques collectifs ou dommages catastropliques, ${ }^{24}$ qui sont capables de miner la confiance du consommateur. Les dommages corporels et les dommages moraux ne sont pas vraiment réparables avec les indemnisations monétaires. ${ }^{25}$

\footnotetext{
18 Voir Dok. 351/76 de 9.9.1976, deuxième version. Dok (79) 415, de 26.9 .1979 et demière version de 25.06.1985 in SCHMIDT SALZER, Joachim. Komtnentar EG-Richtinie Produkthaftung. Verlag, Recht und Wirtschaft, Hejdeberg. Band 1.1986 , p.34ss. Voir sur les travaux plus récents de l'OECD/ OCDE à cause des OGM, MALJEAN-DUBOIS, p. 949 s.

: MARKOWITZ, p. 170ss.

${ }^{20}$ Voir sur l'influence du droit américain sur le droit européen de la responsabilité du fait des produits, MARKOVITZ, Yvan, La directive C.E.E. du 25 juillet 1985 sur la responsabilité du fait des produits défectueux, LGDJ, 1997, p. 32ss.

2: Voir TAYLOR, Simon, L'Harmonisation communautaire de la responsabilité du fait des produits défectueux- Étude comparative du Droit anglais et du droit français, LGDJ, Paris, 1999, p. 54ss.

${ }^{22}$ Voir LANNI, Sabrina, ll modello brasiliano di responsabilità per danni al consomatore, in Rivista di Diritto Civile, ano XLVIII, nr. 2 marzomaprile, 2002, p. 300ss. (p. 281-323) et LIMA MARQUES, Claudia, Contratos no Código de Defesa do Consumidor, 4.ed., Ed. Revista dos Tribunais, São Paulo, 2003, p. 1024ss.

${ }^{23}$ Selon Larroumet, "la fonction préventive d'une responsabilité aggravée ne reposant pas sur la faute est plus que négligeable", LARROUMET, Christian, Réflexion sur la conyention européenne et la proposition de directive de Bruxelles concemant la responsabilité du fait des produits, Recueil Dalloz, 1978, Chron, p. 221.

${ }^{26}$ Sur les dommages qui attentent au même temps contre l'environement et contre les droits des consommateurs, voir BARACHO, José Alfredo de Oliveira Jr., Responsabilidade civil por dano ao meio ambiente, Del Rey, Belo Horizonte, 2000, p. 318.

${ }^{25}$ Voir CADUET, Loï, Les métamorphoses du préjudice, in Les métamorploses de la responsabilités, Sixièmes Journées René Savatier, Potiers, 15 et 16 mai 1997, PUF, Paris, 1997, p. 39ss.
} 
Il faut pourtant repenser les instruments du droit et tenforcer la prévention. La prévention commence par l'information du consommateur, en permettant un choix rationnel et libre. En matière d'aliments et de denrées alimentaires la doctrine a considéré qu'il y a un devoir général d'information des professionnels sur les caractéristiques des produirs, naturels ou industriels. ${ }^{26} \mathrm{La}$ Résolution sur les Droites du Consommateur de l'ONU de 1985 (Res. AG/ONU 20/248, 16.04.1985) a déjà relevé l'importance du droit de l'information au consommateur. ${ }^{27}$ L'obligation des professionnels d'informer le consommateur de façon claire et complète, ${ }^{28}$ incluet certainement l'obligation d'indiquer si le produit alimentaise est génétiquement modifié ou produit à partir d'éléments OGM ou d'animaux nourris aux OGM.

La Directive 2001/18/CE, concernant la traçabilité et l'ériquetage des organismes génétiquement modifiés et la traçabilité des produits destinés à l'alimentations humaine ou animale produits à partir d'organismes génétiquement modifiés, assure l'information des consommateurs européens par un étiquetage spécial s'il y a plus de $1 \%$ d' OGM dans les produits. En décembre 2002, le Parlement européen et le Conseil ont considéré qu'il fallait établir des règles plus strictes $(0,9 \%$ des $O G M$ ) et imposer aussi l'obligation aux importateurs. ${ }^{29}$ Depuis la Conférence ministérielle de l'OMC à Doha du 14 novembre 2001, les gouvernements veulent en outre imposer le principe de précaution au commerce international des produits alimentaires. ${ }^{39}$ Les lignes de protection de l'environnement en matière d'OGM ont été données par le Protocole de Carthagène sur la biosécurité, annexé à la Convention sur la biodiversité biologique. ${ }^{31}$ Les efforts de la communauté internationale et son

26 CALAIS-AULOY, Jean, Droit de la Consommation, 3.ed, Dalloz, Paris, 1992, p. 38 ss.

27 Voir NETTO LOBO, paulo Luiz, A informação como direito fundamental do consumidor, in Revista de Direito do Consumidor (São Paulo), vol. 37, jan/mar.,2001, p. 59ss.

28 Voir sur l'obligation d'informer complètement, BONJEAN, Bernard, Le droit à l'information du consommateur, in LOUSSOUARN, Yvon et LAGARDE, Paul, L'information en Droit PrivéTravaux de la Conférence d'agrégation, LGDJ, Paris, 1978, p. $356 \mathrm{ss.}$

${ }^{29}$ Voir www vistaverde.de/news, 10.12.2002. La proposition de règlement, COM/2002/0515final COD 2001/0180, JO C 331 E du 31.12.2002, p. 30B.312.

34 Voir les critiques à l'Accord sur l'application des mesures sanitaires et phytosanitaires de l'OMC, que ne contient le principe de la précaution, in NOIVILLE, Christine, Principe de précaution et Organisation mondiale du commercelle cas du commerce alimentaire, in journal du Droit International, vol, 2, avr./juin, 2000, p. 266s. Voir sur la biosécurité et le droit de l'OMC, trés critique, MALJEAN-DUBOIS, p. 975 ss.

"Voir MALJEAN-DUBOIS, p.980s et préambule n. 13 de la Directive 2001/18/CEE, JOCEL 106/1-2. 
Codex Alimentarius n'ont pas abouti à un consensus mondial sur le problème ${ }^{32}$. Interdire les larges plantations d'OGM aux Etats-Unis et en Argentine est presque impossible. L'expansion du commerce international est aidée par les décisions de l'OMC et de plus, la liberté de circulation des marchandises connaît très peu d'exceptions. ${ }^{33}$ Il est presque certain que la consommation des OGM sera bientôt un phénomène "global" ${ }^{34}$

Mon hypothèse de travail est que les défis proposés aujourd'hui par l'expansion mondiale du commerce des OGM sur les marchés de consommation sont presque les mêmes que ceux des produits défectueux dans les années 70 , à la différence que maintenant il faut utiliser le principe de la prévention aggravée ou le principe de précaution comme un nouveau concept de droit de la consommation pour les risques alimentaires. ${ }^{35}$ Comme l'affirme Noiville, le cas du commerce alimentaire est particulier en raison des risques directs pour la santé humaine: “...en cas de risque potentiel pour la santé, la précaution postule d'agir, sans attendre que l'existence de ce risque soit confirmée par la preuve scientifique. L'objectif est de dépasser la logique classique exprimée à travers le principe de prévention, inscrit de longue date dans notre droit positif, au profit d'une nouvelle culture du risque. Dans la logique classique, seul un risque avéré justifie que l'on adopte des mesures de prudence: c'est seulement une fois le dommage produit ou rendu fortement probable qu'il devient légitime de chercher les moyens de le cantonner et de prézenir sa réalisation. L'ambition du principe de précaution est précisément d'inverser cette proposition. Le doute, l'incertitude sur la sécurité d'un produit, produisent désormais des effets juridiques et justifient l'adoption de mesures protectrices." 36

\footnotetext{
32. Voir NOIVHLLE, p. $274 \mathrm{~s}$.

35 Voir l'article 8.1 di TRIPS qui autorise d'exceptions pour la protection de la santé publique, in ROTT, Peter, TRIPS-Abkommen, Menschenrechte, Sozialpolitik und Entwicklungständer, in GRURINt. 2/2003, p. 105.

34 Voir texte critique de l'IDEC, Os transgenicos e os riscos à saúde, in www.idec.org.br. Avec l'opinion que les $O G M$ sont déjà un problème mondial et en demandant cohérence du droit international, voir MALJEAN-DUBOIS, p. 949s. et p. 996.

${ }_{35}$ Voir sur la polémique au tour du principe de précaution méme en droit de l'environnement, GRANET, Marie Béatrice, Principe de précattion et risques d'origine nucléaire: quelle protection pour lenvironnement?, in Journal du Droit Intemational, a. 3, jul/sept., 2001, p. 757 s.

${ }^{36}$ NOIVILLF, p. 269.
} 
Ce travail a pour but de vérifier si les lois brésiliennes actuelles sont suffisantes et efficaces pour assurer la prévention des dommages causées par des OGM et la protection de la sécurité des consommateurs. Il faut analyser, dans la première partie, si la législation concernant l'information des consommateurs et le contrôle de l'obligation d'informer, au Brésil et dans le Mercosur, est suffisant pour assurer une véritable prévention des dommages et efficace pour assurer les bases de prévention efficace des dommage causés par des OGM et, dans la deuxième partie, il faut établir quelles sont les base de l'obligation de sécurité en matière d'OGM et pour la réparation des accidents de consommation causées par des OGM, vérifiant si la législation sur la responsabilité civile et le droit international privé du fait de produits avec OGM est suffisante et efficace.

\section{I - Prévention des dommages causés par des OGM: la législation concernant l'information aux consommateurs et le contrôle de l'obligation d'informer}

Si mon hypothèse de travail est correcte, les premières années du XXI siècle sont semblables aux années 70 du siècle passé, lorsque les légistations internes des pays n'étaient pas suffisantes et assez efficientes pour prévenir ct combattre les dommages causés pour des produits défectueux. Il a fallu attendre les initiatives des organismes internationaux et d'intégration pour que la législation nationale connaisse une évolution sensible.

Aujourd'hui la doctrine considère qu'il y a un devoir général de sécurité pour les produits, naturels ou industriels, et les services fournis sur les marchés de consommation. Les instruments utilisés pour prévenir les risques sont le contrôle de l'Etat au moyen d"evaluation préalables et aurorisations de mise sur le marché, et la transparence au moyen d'une politique d'information aux consommateurs, échanges d'informations par les autorités compétentes et surveillance des dangers. Nous nous concentrerons dans cet article sur la question de la transparence. ${ }^{37}$

La doctrine considère qu'il y a un devoir général d'information des professionnels sur les caractéristiques er risques des produits, naturels ou industriels. ${ }^{38}$ 11 y a aussi un droit d'information des consommateurs, assuré par la Résolution sur les Droits du Consommateur de l'ONU de 1985 (Res. AG/ONU 20/248, 16.04.1985). ${ }^{39}$

\footnotetext{
${ }^{37}$ TOMASETTI. Alcides $\int \mathrm{r}$, O objetivo de transparência e o regime jurídico dos deveres e riscos de informação nas declaraçôes negociais para corsumo, in Revista de Direito do Consumidor, vol. 4, 1992 , p. 52.

3 CALAIS-AULOY,p. 38 ss.

39 Voir NETTO LOBO, p. 59 ss.
} 
L'obligation des professionnels d'informer le consommateur d'une façon claire et complète $\mathrm{s}^{40}$ doit inclure l'information sur la présence des OGM.

Mais on peut aller plus loin. Comme la science n'a pas encore établi d'une manière sûre que les OGM n'ont pas d'effets néfastes sur les consommateurs ('théorie de l'évaluation divergente des risques' de Rudolf Streinz), ${ }^{41}$ l'information sur la présence des OGM est une information sensible, cela veut dire quelle est une information nécessaite pour éviter les risques et pour exprimer la volonté d'achat des consommateurs, en rant que personne humaine rationnelle, libre et digne.

D'un autre côté, l'obligation d'informet relative aux composants, aux caractéristiques er l'origine d'un produit ou aliment est présente dans presque toutes les législations de protection du consommateur du monde. De même les droits fondamentaux, et notamment celui de la dignité de la personne humaine, sont cités dans les Constitutions nationales. Les règles de l'ONU, les règles constiturionnelles et les règles de droit comparé de la consommation peuvenr inciter la doctrine consumériste à défendre l'idée d'un droit fondamental aux informations nécessaires à la santé et à la dignité humaine.

Les droits fondamentaux, comme l'affirme Roberr Alexy ${ }^{42}$, ne sont pas seulement des droits subjectifs des personnes (subjektive Rechte), mais des normes présentes dans l'ordte juridique (objektive Normen). Ces normes sont en vigueur sous une forme spéciale (Geltung), car elles imposent un ordre objectif des valeurs (objektive Wertordnung), un système de valeurs à tout le Droit (Wertsystem), elles représentent les grandes décisions de la Constitution pour routes les disciplines du Droit (verfassungsrecbtliche Grundentscheidung für alle Bereiche des Rechts): droit privé, droit économique, droit de la consommation et pas seulement pour le droit administratif. ${ }^{43} \mathrm{Si}$ on pouvait établir l'existence d'un droit fondamental ou droit humain à l'informarion nécessaire à la santé et dignité humaine, ce

\footnotetext{
40ir sur l'obligation d'informer complètement, BONJEAN, Bernard, Le droit à l'information du consonmateur, in LOUSSOUARN, Yvon et LAGARDT, Paul, L'information en Droit Privé- Travaux de la Conférence d'agrégation, LGDJ, Paris, 1978, p. 356ss.

"Voir KNORR, p. 90 ss.

4? ALEXY, Robert,

Recht, Vernunft, Diskurs - Studien zur Rechtsphiłosphie,

Ed. Suhrkamp, 6.Reimp.,Frankfurt, 2000, p. 262.

43. Voir SARLET, Ingo, Os direitos fundamentais sociais na Constituição de 1988 , in

Revista de Direito do Consumidor vol. 30, p. 98.
} 
droit fondamental serait une directive (Richtlinien) et une véritable signal (Impulse) à l'attention des Etats pour organiser (Rechte auf positive Handlugen) ${ }^{44}$ les marchés de distribution et de consommation et assurer le droit d'information sur les OGM aux consommateurs. ${ }^{45}$

Voyons maitenant la situation dans la législation nationale, en choisissant le Brésil et le Mercosur, comme exemples des défis que les OGM proposent au droit de la consommation. ${ }^{46}$

\section{A) La législation nationale sur l'information des consommateurs et le contrôle de l'obligation d'informer: les cas brésilien}

Informer les consommateurs, c'est prévenir les dommages. Prévenir, c'est aussi contrôler les fabricants, les fournisseurs des produits agricoles, les importateurs, les paysans sur la prestation de l'information. Dans la prévention d'un dommage il faut considérer la vulnérabilité du consommateur, cela veut dire la 'fragilité' de sa position et 'la nécessité de sa protection' en tant que personne humaine. ${ }^{47}$

Le cas brésilien peu servir d'exemple. D'un côté, le pays a une société civile assez engagée dans la lutte pour ses droits de consommateurs et pour la protection de l'environnement. L'Association IDEC, le Greenpeace et le Forum des Associations de Défenses du Consommateurs ${ }^{48}$ ont organisé dès 2000 la campagne "Pour un Brésil

${ }^{4}$ Selon ALEXY, Robert. Theorie der Grundrechte. Frankfurt: Suhrkamp, 1996, p. 179.

${ }^{45}$ Selon ALEXY/Studier, p. 262: "Gesetzgebung, Verwaltung und Rechstprechung empfangen von ihm Richtlinien und Impulse'."

${ }^{46}$ Le choix du Brésil n'est pas seulement à cause de l'origine de l'auteur, mais aussi à cause de l'intérêt qui le pays présent en matière de protection de protection de l'envitonnenent (voir Benjanin, Antono, Einführung in das brasilianische Unweltrecht, in Das Recht der lusophanen Länder, JAYME, Erik (Ed.), Nomos Verlag, Baden.Baden, 2000,p. 105ss). Le Brésil fait partie du Mercosur et ses principales partnets d'importation ex exportation son les États-Unis et 1 Argentine, deux pays où la production d'OGM est assez commun, on affirme même que $80 \%$ de la production de soja et maïs de l'Argentine est des OGM, voir www.idec.org.br.

"COHET-CORDEY, Frédérique, "Préface", in Vulnérabilité et Droit- Le développenent de la vulaérabilité et ses enjeux en droit, Cohet-Cordey, Frédérique (Org), Presses Universitaires de Gremoble, Grenoble, 2000, p. 9. Voir aussi LOPEZ CABANA, Roberto, Defesa juridica de los más débiles, in Revista de Direito do Consumidor, vol.28, 1998, p. 21

4V Voir aussi la manifestation de tous les Commissions adninistratifs de protection des consommateus du Brésil, les PROCONs, in Monção sobte alimentos transgênicos-22a Reunião nacional dos PROCONS estaduais t municipais das capitais, Revista de Direito do Consumidor n.30, p. 269-70 
Libre des OGM" ("Por um Brasil Livre de Transgênicos"). ${ }^{49}$ Le Ministère Publique exerce cette fonction de protection des consommateurs et de l'environnement. ${ }^{50}$ D'un autre côté, l'Etat a créé une Commission de Sécurité Biogénétique (dénommée CTNBio), ${ }^{\text {,t }}$ un organe de législation de protection de l'environnement (dénommé CONAMA), un organe de contrôle de l'autorisation de mise sur le marché des OGM ou de dissémination volontaire des OGM dans l'environnement (dénommé IBAMA), ${ }^{52}$ et on a assuté aux consommateur un droit général d'information sur tous les composants des aliments et de tous les produits (Art. 6,I,11,III, 7, 9, 12,14,18,20,30 et 31 du Code Brésilien de la Défense du Consommateur, Loi 8.078/90) et un Décret spécial sur l'étiquetage des OGM au Brésil ${ }^{53}$ Voyons ce qui s'est passé dans la "law in action".

L'entreprise Montsanto a demandé en 1998 à la Commission brésilienne de Biosécurité une 'dé-réglementation' de la soya roundy up, pesticide-tésistent. La commission a cédé aux pressions 14 jours après la demande et a autorisé la plantation au Brésil de cette espèce d'OGM. ${ }^{54}$ L'association de défense du consommateur-IDEC, de São Paulo, a demandé judiciairement une suspension de cette autorisation administracive, car il manquait l'étude d'impact de l'environnement et, à l'époque, la réglementation sur l'information impérative aux consommateurs..$^{55}$ Selon la Constitution brésilienne (Art. 225, $\$ 1^{\circ}$, IV , de la Constitution Fédérale Brésilienne de 1988) et le droit infra-constitutionnel (Loi 6.839/81, Art. 14 et 16) il est nécessaire d'élaboré une telle étude avant. de libérer des organismes génétiq̨uement modifié dans la biodiversité brésilienne. ${ }^{56}$ Le juge de première instance, en utilisant le principe de

\footnotetext{
49. Voir site de l'IDEC, www.idec.org.br .

5n Voir BENJAMIN, Antônio Herman, Um novo modelo para o ministério público na proteção do meio ambiente, Revista de Direito Ambiental (Säo Paulo), vol. 10, abr/jun. 1998, p. 7ss.

51 CTNBIO-Comissão Técnica Nacional de Biossegurança, créé par le Decreto 1.752, du 20.12.1995, voir LAZZARINI, Andrea et leFEVRE GUIMARÃES, flávia, Alimentos Transgênicos, in Revista de Dircito do Consumidor, vol. 33, jan/mar., 2000, p. 203.

52. Voir La Résolution 305, de 12.06.2002 sur la licence des organismes génétiquement modifiées du CONAMA autorise le IBAMA, à côté de la CTNBIO, a réaliser cette tache administrative (Resolução $\mathrm{n}^{\prime \prime} 305$, de 12 de junho de 2002, dispõe sobre Licenciamento Ambiental, Estudo de Impacto Ambiental e Relatório de Impacto no Meio Ambiente de atividades e empreendimentos com Organismos Geneticamente Modificados e seus derivados).

5.3 Décret 3871, du 18.07.2001, in Revista de Direito do Consumidor, vol. 40, out/dez, 2001, p. 286 288.

${ }^{54}$ La demande était du 15.06.1998 et la libération du 29.06.1998, voir LAZZARINI/LEFEVRE, p. 203. 55 Voir les matériaux pour l'action in Revista de Direito do Consumidor, vol. 40, out/dez., 2001, p. 233-241. 56 ROCHA, Maria Isabel de Matos, Reparação de danos ambientais, Revista de Direito Ambiental, vol. 19 (jul/set. 2000$)$, p.150-151
} 
précaution, a ordonné la suspension de l'autorisation. ${ }^{57}$ Le Tribunal Régional Fédéral a maintenu la décision de première instance et a interdit la commercialisation de la soya roundy up sur tout le territoire brésilien en $2000 .^{58}$

57 Voir sentence au Procès 1998.34.00.027682-0, 6 Vara Seção Judiciária do Distrito Federal, Brasília (DF), j. 26.06. 2000, Juge Antônio Souza Prudente: "No mérito, a presente ação procede, in totum, garantindo-se o resultado útil deste processo, pela eficácia da tutela cautelar já deferida por este Juízo, nos autos do processo n1 98.34.00.276681-8, com natureza mandamental-inibitória e que, ora, se subsume e fica absorvida pela eficácia que resultaxá deste ato sentencial de mérito.Quanto à exigência de realização de prévio Estudo de Impacto Ambiental da MONSANTO e de todos os outros pedidos à CTNBio formulados, nesse sentido, adoto, ainda, aqui, as razões expendidas pelo ilustre Representante do Ministério Público Federal, Dr. Aur

élio Veiga Rios, nos autos do processo cautelar referido, nestas letras: O EX não é una formalidade de menos; uma faculdade, arbitrio ou capricho que possa ser dispensada no exame tão delicado das conseqüencias do descarte de $O G M$ no meio ambiente. A exigência constitucional não pode ser, evidentemente, limitada por um decreto regulamentado. $O$ art. 225, inciso IV, da Constituição Federal exige, na forma da lei, estudo prévio de impacto ambiental, para instalação de qualquer obra ou atividade potenciamente causadora de significativa degradação do meio ambiente, incluindo se nesse rol a liberação de organismo geneticamente modificado.

Nos termos da Lei n1 6.938/81 e da Resolução n1 237, de 19/12/97, do Conselho nacional do Meio Ambiente (CONAMA), que expressamente exige a licença ambiental en casos de introdução de espécies geneticamente modificadas no meio ambiente".

58 Voir décision du Tribunal Regional Federal, $1^{2}$ Região, APC 2000.01.00.014661-1/DF, j. 08.08.2000, Juge Assusete Magalhães:"

CONSTITUCIONAL E PROCESSUAL CIVIL - AÇÃO CAUTELAR - LXBERACCÃO DO PLANTIO E COMERCIALIZAÇÃO DE SOJA GENETICAMENTE MODIFICADA (SOJA ROUND UP READY), SEM O PREVIO ESTUDO DE IMPACTO AMBIENTAL - ART. 225, $\$$ $1^{\circ}$, IV, DA CF $/ 88 \mathrm{C} / \mathrm{C}$ ARTS. $3^{\circ}, 9^{\circ}$ E $10^{\circ}, \$ 4^{\circ}$, DA LEY $N^{\circ} 6.938 / 81$ E ARTS. $1^{\circ}, 2^{\circ}$, CAPUT E $51^{\circ}, 3^{\circ}, 4^{\circ}$ E ANEXO I, DA RESOLUCÄO CONAMA N ${ }^{\circ} 237 / 97$.

- INEXISTENCIA DE NORMAS REGULAMENTADORAS QUANTO À LIBERAÇÃO E DESCARTE, NO MEIO AMBYENTE, DE OGM.

- pRINCÍPIOS DA PRECAUÇÃo E DA INSTRUMENTALIDADE DO PROCESSO CAUTELAR

- PRESENGA DO FUMUS BONI IURIS E DO PERICULUM IN MORA

- poder geral De CAUTEla dO MAGistrado

- INEXISTENCCIA DE JULGAMENTO EXTRA PETITA

- ART. 808, III, DO CPC

- INTELIGENCIA. ...IV

-O art. 225 da CF/88 erigiu o meio ambiente ecologicamente equilibrado ,a bem de uso comum do povo e essencial à sadia qualidade de viła, impondo-se ao Poder Público e à coletividade o dever de delendê-to e preservá-lo para as presentes e futuras geraçoes", incumbindo ao Poder Público, para assegurar a efetividade desse direito, exigir, na forma da lei, para instalaçāo de obra ou atividade potencialmente causadora de significativa degradação do meio ambiente, estudo prévio de impacto ambiental, a que se dará publicidade" (art. $225, \$ 1^{\circ}, \mathrm{W}$, da CF/88), V - A existência do fumus boni iuris ou da probabilidade de tutela, no processo principal, do direito material invocado, encontra-se demonstrada especialmente: a) pelas disposições dos arts. $8^{\circ}, 9^{\circ}$ e $10^{\circ}, \$ 4^{\circ}$, da Lei $0^{\circ} 6.938$, de $31 /$ $0 B / B 1$ - recepcionada pela $C F / 88$ - e dos arts. $1^{\circ}, 2^{\circ}$, caput e $\$ 1^{\circ}, 3^{\circ}, 4^{\circ}$ e Anexo 1 da Resolução CONAMA $n^{\circ} 237 / 97$, à luz das quais se infere que a definição de ,obra ou atividade potencialmente causadora de significativa degradaçào do meio ambiente", a que se refere o art. $225, \$ 1^{\circ}$, IV, da CF/ 88, compreende „a introdução de espécies exóticas e/ou geneticamente modificadas", tal como 
Notre Institut, l'Institut Brésilien de Politique et Droit du Consommateur (Brazilconsumer), qui est une organisation scientifique sur le Droit de la Consommation a prévu, lui aussí, une résolution sur le droit à l'information et de sécurité des consommateurs en 2000: "Pour assurer la protection des droits basics $d u$ consommateur prévue au Code de Protection du consommateur (Loi 8.078/90) et la protection de l'environnement prévue dans la législation spécifique, il est nécessaire de réglementer les espèces génétiquement modifiées, tout particulièrement en ce qui concerne la sécurité alimentaire, l'étiquetage plein de tous les produits (industriels et in natura), ainsi que la réalisation des études d'impact dans l'environnement préalablement à la libération de chaque OGM dans l'environnement. Jusqu'a l'élaboration de la réglementation et des évaluations des risques pour le pouvoir public, soit sur l'impact sur la santébumaine (selon

consta do Anexo $X$ da aludida Resolução CONAMA $n^{\circ} 237 / 97$, para a qual, por via de consequêencia, necessário o estudo prévio de impacto ambiental; b) pela relevância da tese de que o parecer conclusivo da CTNBio não tem o condào de dispensar o prévio estudo de impacto ambiental, pata o plantio, em escala comercial, e a comercializaçäo de sementes de soja geneticamente modificadas, especialmente ante séria dúvida quanto à constitucionalidade do art. $2^{\circ}$, XIV, do Decreto $\mathrm{n}^{\circ} 1.752 /$ 95, que permite à CTNBio dispensar o prévio estudo de impacto ambiental - de competência do IBAMA - em se tratando de liberação de otganismos geneticamente modificados, no neio ambiente, em face do veto presidencial à disposição constante do projeto da Lei $n^{\circ} 8.974 / 95$, que veiculava idêntica faculdade outorgada à CTNBio. Precedente do STF (ADin $\Omega^{\circ} 1.086-7 /$ SC, Rel, Min. Ilmar Galvão, in DJU de 16/09/94, pág. 24.279); c) pela vedação contuda no art. $8^{\circ}$, VI, da Lei $n^{\circ} 8.974 / 95$, diante da qual se conclui que a CTNBio deve expedir, previamente, a regulamentação relativa à liberação e descarte, no meio ambiente, de organismos geneticamente modificados, sob pena de se tornarem ineficazes outras disposiçōes daquele diploma legal, pelo que, à míngua de normas regulamentadoras a respeito do assunto, até o momento ptesente, juridicamente relevante é a tese de impossibilidade de autorização de qualquer atividade relativa à introdução de OGM no meio ambiente; d) pelas disposiçóes dos arts. $8^{\circ}$, Vx, e $13, \mathrm{~V}$, da Lei $\mathrm{r}^{\circ} 8.974 / 95$, que sinalizam a potencialidade lesiva de atividade cujo descarte ou lberação de $O G M$, no meio ambiente, sem a observância das devidas cautelas regulatnentares, pode causar, desde incapacidade para as ocupaçōes habituais por mais de 30 dias e lesão corporal grave, até a morte, lesão ao meio ambiente e lesão grave ao meio ambiente, tal como previsto no art. $13, \int S 1^{\circ}$ a $3^{\prime \prime}$, da Lei $n^{\circ} 8.974 / 95$, tipificando-se tais condutas como crimes e impondo-thes severas penas. VX - A existència de uma situação de petigo recomenda a tucela cautelar, no intuito de se evitar - em homenagern aos princípios da precaução e da instruntentalidade do processo cautelar - , até o deslinde da ação principal, o risco de dano irreversivel e irreparável ao meio ambiente e à saúde pública, pela utilização de engenharia genética no meio ambiente e em produtos alimentícios, sem a adoção de rigorosos critérios de segurança.VII

- Homologaça do pedido de desistencia do XBAMA para figurar no polo ativo da lide, em face da superveniencia da Medida Provisória n" 1.984-18, de 01/06/2000. VIII -.. Preliminares rejeitadas. Apelaçóes e remessa oficial, tida como interposta, improvidas." 
l'exemple de celles qui sont faites pour les médicaments), soit dans l'environnement, aucune espèce d'organisme génétiquement modifié ne doit être autorisé au Brésil."

Un an plus tard, en 2001, le gouvernement brésilien a élaboré la réglementation d'emballage et d'information aux consommateurs sur les aliments qui contiennent des OGM ou des composants OGM, à savoir le Décret 3.871, du 18 juillet 2001. 60 Selon ce Décret présidentiel, l'obligation d'informer les consommateurs est de rigueur seulement si les composants du produit contiennent chacun plus de $4 \%$ d'OGM. ${ }^{6}$ Ansi, si une soupe ou un aliment pour enfants est faite avec du maïs et du soja génétiquement modifiés, chaque ingrédient peut avoir jusqu'à $3,9 \%$, soit un total de $7,89 \%$ d'OGM sans obligation d'informer le consommateur brésilien. De nouveau l'Association de défense du consommateur-IDEC, de São Paulo, a demandé judiciairement le prononcé de l'inconstitutionnalité du Décret, car la Constitution brésilienne prévoit parmi les droit fondamentaux la protection des intérêts des consommateurs, et estipule que les droits des consommateurs sont des limites à la libre initiative (Art. 5,XXXIl et Art. 170,V de la CF $/ 88)^{62}$. L'IDEC a attaqué le Décret lui-même pour violation du Code Brésilien de la Consommation, qui prévoit le droit à l'information aux articles 6\%, I, II, III, 9 et 31 de la Loi $8.078 / 90$ et de la Résolution 30/248 de l'Assemblé Générale des Nations Unies, de 16.04 .85 (Art.3). ${ }^{63}$

59 Conclusion nr. 6 du "V Congresso Brasileiro de Direito do Consumidor, Beto Forizonte, 02.05.2000, Painel 10 - Direito Básico à Segurança", in Revista de Direito do Consumidor (São Paulo), vol. 35 , jul/set. 2000, p. 262. Dans l'original: "6. Visando assegurar a proteção dos direitos básicos do consumidor previstos no CDC e a proteção ambiental prevista na legislação específica, sustentamos a necessidade de regulamentação adequada das espécies transgênicas, especialmente ao tocante à segurança dos alimentos, à rotulagem plena de todos os produtos (embalados, in natura e todos os demais) bem como a realizaçāo do estudo de impacto ambiental prévio a cada liberaçăo de ONG's no meio ambiente. (aprovada por maioria) Até que a adequada regulamentação seja adotada e as avaliaçoes rigorosas de risco sejam executadas pelo Poder Público, tanto no tocante aos impactos à saúde humana (a exemplo do que é feito com o medicamento), como ambiental, nerhuma espécie transgênica deve ser autorizada no país. (aprovada por maioria)."

${ }^{50}$ Décret 3.871, du 18 juillet 2001, "Disciplina a rotulagem de alimentos embalados que contenham ou sejam produzidos com organismo geneticamente modificados, e dá outras providências (DOU 19.06.2001)", in Revista de Direito do Consumidor, vol, 40, out/dez., 2001, p. 286-288.

b? Post Scriptum: Voir réduction à $1 \%$ ex vi Decreto $4.680 / 2003$ "sobre o direito de infornação dos consumidores, rotulagem e símbolos especiais para alimentos e ingredientes alimentares destinados ao consumo humano ou animal" in www planalto.gov.br.

62 Voir FRETTAS, Vladimir Passos de, A Corstituição Federal e a efetividade das normas ambientais, Editora Revista dos Tribunais, São Paulo, 2000, p. 17/s.

6. Voir l'action de l'LDEC et du MPF, in Revista de Direito do Consumidor, vol, 40, out/dez. 2001, p. 233-241. 
La question est encore pendante, mais le gouvernement brésilien n'a jamais contrôlé l'étiquetage des produits OGM au Brésil. En 2002, l'WDEC avec l'aide d'un institut suisse, a testé 36 produits de Supermarché à São Paulo et a décelé des OGM dans 11 produits. Aucun de ces produits ne présentait une information aux consommateurs.

En mars 2003, en raison de la pression des producteurs agricoles, même si la décision judiciaire prohibant la plantation des OGM au Brésil teste toujours valable, le gouvetnement brésilien a autorisé à la vente de ${ }^{64}$ la récolte 2003 de soja OGM!, en tant que composants de produits d'originne brésilienne!

La nouvelle norme, avec un peu d'ironie, permet la 'vente' des OGM brésiliens comme produits dérivés et interdit leur usage comme 'semence', en visant l'extinction des OGM jusqu'au 31 janvier 2004 (Art.1,\$1 MP 113/2003). L'Etat brésilien, selon l'article $1, \$ 3^{\circ}$ de cette norme, doit aussi essayer des formules pour 'exporter' vers d'autres consommateurs étrangers ces produits-OGM et éviter que la "récolte OGM" soit consommée au Brésil ! La règle prévoit l'étiquetage impératif de tous les produits dérivés ou en utilisant des OGM (Art. $2^{\circ}$ ) et la création d'une certification négative: "Sans OGM" $\left(\Lambda\right.$ rt. $\left.3^{\circ}\right)$. La norme impose des sanctions administratives lourdes en cas de violation. On peut seulement espérer que cette norme aura le résultat escompté : contrôler les OGM au Brésil !

L'exemple brésilien démontre que les décisions d'interdiction judiciaires éparses, les normes administratives et la normalisation abstraite d'un droit à l' information des consommateurs ne sont pas suffisantes. Il faut un contrôle administratif lourd et puissant. Il faut une société civile, avec des associations de défense du consommateur et un Ministère Public actifs, mais surtout il faut développer des instruments juridiques efficaces, pour la répression des fraudes d'information aux consommateurs. Le droit à l'information des consommateurs ne doir rencontrer aucune limite autre que celle de la détermination scientifique de la présence de l'OGM. Ainsi, l'autorisation des OGM jusqu'à $4 \%$ pour chaque composant d'un produit brésilien viole fortement le droit à l'information. Il y a plusieurs colorants et ingrédients qui ont un pourcentage inférieur et qui sont mentionnés sur l'étiquetage. Si vraiment les OGM ne causent pas de dommages à la santé publique, alors il n'y a aucune raison de ne pas informer les consommateurs. L'information sur les OGM est une information nécessaire à la santé et la dignité humaine. La loi brésilienne doit être modifiée et le gouvernent doit commencer un contrôle efficace, avec de lourdes sanctions administratives (et même pénales) en cas de violation des règlements.

\footnotetext{
${ }^{64}$ La 'Medida Provisória' 113, du 26.03.2003, "estabelece normas para a comercialização da produção de soja da safra de 2003", DOU 27.03.2003. (Post Scriptun: Voir Lei 10.688, da 13.06.2003 in www.planalto.gov.br).
} 


\section{B) Le cas du Mercosul comparé à celui de l'Union Européenne}

Le droit de la consommation a une vocation internationale. ${ }^{65}$ En théorie, le consommateur ne saurait être prétérité sur le plan de la sécurité, de la garantie ou de l'information parce qu'il achète un produit ou un aliment provenant d'un autre pays ou fourni par une entreprise étrangère. Notamment, s'il y a un projet de rapprochement économique entre les pays, comme la formation future d'une marché commun ou d'une union douanière, cette protection sans frontière du consommateur doit être un des objectifs de cette intégration économique. Les Communautés Européennes ont beaucoup légiféré en matière de protection du consommateur et de la concurrence. ${ }^{66}$ En revanche, le Mercosur (Marché Commun du Sud), jeune projet d'intégration économique entre Argentine, Brésil, Paraguay et Uruguay (et avec les pays associés, Chili et Bolivie) ${ }^{67}$, fondé par le Traité d'Asunción du 26 mars 1991, ${ }^{68}$ n'a pas téussia a organiser un Règlement standard minimal de protection des consommateurs dans la région ${ }^{69}$ A ce jour, reste en vigueur la Résolution $126 / 94$, selon laquelle il faut appliquer

${ }^{65}$ Voir BOURGOIGNIE, Thierry, Elements pour une théorie du droit de la consommation, 1988, P.215ss.

(t) Cf. KRÄMER, Ludwig, La CEE et la protection du consommateur, Collection Droit et Consommation 15, Story, Bruxelles, 1988, S. 377.

${ }^{67}$ Le Mercosur a été crée dans le cadre de l'ALADI-Asociación Lationoamericana de lntegración, fondée en 1980 par le Traité de Montevideo, voir SALOMÃO Filho, Calixto et SAMTLEBEN, Jürgen, Der Südamerikanische Gemeisame Markt - Eine rechtliche Analyse des Mercosur - Teil 1, WM, 33 (1345.1352), 1992, p. 1346 et DROMI, Roberto, EKMEKDJIAN, Miguel A., RIVERA, Julio C., Derecho Comunitario-Sistemas de Integración Regimen del Mercosur, Ed. Ciudad Argentina, Buenos Aires, 1995, p. 25ss.

os "Tratado para la constitución de un Mercado Común entre la República Argentina, la República Federativa del Brasil, la República del Paraguay y la República Oriental del Uruguay", entrée en vigueur le 29 novembre 1991 et "Acuerơ de complementación económica n. 34 celebrado entre los gobiernos de los Estados Partes del Mercosur y el Gobierno de la República de Bolivia, Puenta del Este, Uruguay, 7.12.1995, AlADi (ACE 34)" et "Acuerdo de complementación económica n. 34 celebrado entre los gobiernos de los Estados Partes del Mercosur y el Gobierno de la República de Chile, San Luis, Argentina, 25.06.1996, ALADI (ACE 35)". Voir Mercosul: Legislação e Textos Básicos, Comissão Parlamentar Conjunta do Mexcosul-Seção Brasiletra e Ministério das Relaçŏes Exteriores (Ed), Senado Federal, Brasilia, 2000, p. 15ss et ARAÚJO, Nádia, MARQUES, Frederico Magalhăes e REIS, Márcio, Código do Mercosul. Tratados e Legislação, Renovar, Rio de janeiro, 1998, p. 17ss, pour un texte en anglais, voir O'KEEFE, THOMAS A., Latin American Trade AgreementsA5-1 to A5-15, 30 I.L.M. 1041 -1063 (1991).

${ }^{69}$ Voir les efforts pour harmoniser les règles sur la protection du consommateur au sein du Mercosul, in LMA MARQUES, Claudia, A proteção do consumidor: aspectos de direito privado regional e geral, El Derecho Internacional Privado en las Américas (1974-2000), Cursos Je Derecho Intemacional - vol. II (Parte 2), Ed. Secretaria General-Subsectetaria de Asuntos Juridicos/OEA, Washington, 2002, p. 657 - 779 et ARRIGHI, Jean Michel, La protección de los consumidores y el Mercosur, Revista de Direito do Consumidor, São Paulo, 2 (1992), p. 134 ss, STIGLlTZ, Gabriel, El derecho del consumidor en Argentina y en el Mercosur, in Derecho del Consumidor (Buenos Aires), vol. 6, 1995, p. 20ss et TONIOLLO, favier Alberto, La protección internacional del consumidor-Reflexiones desde la perspectiva del Derecho Internacional Privado Argentino, in Revista de Derecho del Mercosur (Buenos Aires/Porto Alegre), ano 2, nr. 6, diciembre de 1998, p. 94-117. 
la loi du pays de commercialisation du produit ou de l'aliment. ${ }^{70}$ Ainsi, si le soja OGM d'Argentine doit entrer au Brésil, l'importateur doit suivre la législation brésilienne et indiquer qu'il y a des OGM dans cet ingrédient. De cette manière, les chocolats qui viennent d'Argentine et contiennent du soja OGM doit l'indiquer sur l'étiquetage en portugais, et ce dès qu'il y a plus de 4\% d'ingrédient OGM dans le produit. Or, en vérité, cette procédure d'information du consommateur brésitien n'a pas eu lieu et même les Associations de Défense du Consommateur ne savent pas dans quel produit national ou importé il y a des OGM au Brésil. La conclusion est alors que la règle du Mercosur est insuffisante pout assurer l'information des consommateurs dans la région.

Au sein du Mercosur, la prévention des dommages est complétée par une série de Résolutions sur la sécurité des produits et d'une Résolurion sur la garantie des produits qui. établit un système d'échange d'information en cas de défaut des produits et une série de Résolutions sur l'étiquełage. ${ }^{71}$ L'étiquetage des OGM ne fait pas encore l'objet d'une Résolution spécifique, la Résolution générale sur le droit de la consommation, Res. 126/94 continuant à être l'unique règle du Mercosur sur l'OGM. ${ }^{72}$

Ici la législation dérivée des Communautés Européennes peut servir de point de comparaison, ${ }^{73}$ La gestion des risques en Europe a trois instruments fondamentaux de prévention des dommages: l'évaluation des risques pour l'environnement, la surveillance et

70 MERCOSUL/GMC/RES. 126/94, in: Boletim de Integração Latino-Anericana/MRE/BR, 15,p. 133.

$"$ Voir les normes sur les emballages au Mercosux, les Res. 10/91, 17/92 sur les emballages des produits industrialisés et des aliments, aussi les Res. $28 / 93,35 / 93,36 / 93,48 / 93$ et $87 / 93$, qui ont été introduit dans la législation brésilienne par des normes spécifiques de 1

'Institut brésilien de Mesure et Normes Techniques, 'Portarias' de l'TNMETRO (DOU 16/04/93, p. 49/99 et DOU 21/9/92, p. 13.160).

72 Voir la législation dérivé du Mercosur en apportant des contributions à la profection de la santé et de la sécurité des consommateurs les Résolution 31/92, 19/93, 46/93 83/93, 91/93 et 55/94 sux les additifs permis dans des aliments, la Res. 19/93 sur les colorants permis, la Res. $56 / 94$ sur les toxines dans le hait et plusteurs types des farines, les Res. 31/93,82/93 et $63 / 94$ sur le types et la qualité du lait, les Res. 69/93,70/93 et $72 / 93$ sur la qualité des fromages et du beurre, la Res. $32 / 92$ et $59 / 93$ règles générales sur la qualités des aliments, les Res. $11 / 93$ et $44 / 93$ règles générales sur les produit animales et la Dec. 06/93 approuve l'accord Sanitaire et Phytosanitaire du Mercosur, la Res. 57/94 sur les résidus toxiques et tnédicaments en produits d'origine animal, comme la viande et les Res. 59/ $94,60 / 94$ et $62 / 94$ sur les mesures phytosanitaires et Res. 64/94, 65/94, 66/94, 67/94 sur les mesures zoo-sanitaires et sur la qualité de la viande, la Res. 04/92 réglés sur la fabrication et qualité des médicaments, la Res. $54 / 92$ règles sur l'harmonisation des normes sur la sécurités de joues pour les enfants et la Res. $58 / 92$ règles sur la commercialisations des produits industrialisé, introduit dans la législation brésilienne par des normes de l'Institur INMETRO. Aussi en matière de protection du consommateur et protection de l'environnement le Mercosur a établie des normes, comme par exemple la Dec. 14/94 sur le transport des produits dangereux, la Res. 80/93 sur les sprays, et les Res. $09 / 91,6 / 92,9 / 91,26 / 93$ sur la sécurité des automobiles et sur les émissions de gaz carboniques. Voir Boletim de Integração Latino-Americana, 12,p. 41ss et $15, \mathrm{p} .93 \mathrm{~s}$.

${ }^{3}$ Voir SCHERBE, Virgínia Amaral da Cunka, O Direito Ambiental no Mercosul, in Revista de Direito Ambiental, vol. 23 (2001), p. 125s. 
le contrôle par la Commission, et la transparence avec information aux importateurs, aux autorités et aux consommateurs.

Le principe qui guide cette législation est celui de la prévention (Art.1, Ditective 2001/ 18/CE, du 12 mars 2001 relative à la dissémination volontaire d'organismes génétiquement modifiés dans l'environnement et abrogeant la directive $90 / 220 / \mathrm{CEE}$ ), visant à protéger les effets négatifs des OGM sur la santé humaine et l'environnement (Art. 4 de la Directive $2001 / 18 / \mathrm{CE}$ ). La législation eutopéenne établit une "évaluation des risques pour l'environnement" (Art. 2 de la Directive 2001/18/CE), une autorisation préalable aux OGM (Art.6 de la Directive) et un système d'échange d'infotmations enrre les autotité compétentes et la Commission, et d'information au public (Art. 11 et Art. 24 de la Directive).

Face aux consommateurs les produits et les éléments de produits OGM doivent suivre une sétie de normes, la Directive 70/457/CEE, Directive 70/458/CEE, Décision 74/234/CEE et le Règlement 89/397/CE, sans préjudice de la principale norme, la Directive 2001/18/CE. L'Union européenne a créé des règles sur l'étiquetage des OGM, "afin de fournir une information claire, sur une étiquette ou un document d'accompagnement' (Art. 26 de la Directive 2001/18/CE). Les Etats-membres, comme l'Allemagne par exemple, ont aussi des lois spécifiques sur les tcchniques génétiques, comme la Gesetz zur Regelung der Gentechnik, du 20 Juni 1990, actualisée le 19 juillet 2002, qui a créé une Commission pour la sécurité biologique (Zentrale Kommission für die Biologische Sicherbeit). ${ }^{74}$

Le système européen peut être résumé par les actions positives des pays membres suivantes: 1) l'autorisation de la mise sur le marché des OGM avec l'évaluation des risques sur l'environnement; 2) le contrôle de l'étiquetage ou information accompagnant pour une quantité d'OGM supérieure à $1 \%$ (ou dans le futur $0,9 \%$ ) dans tous les produits agricoles ou industriels; 3) l'obligation des rapports de surveillance et d'échange d'information; 4) l'obligation de notification aux autorités compétentes des dangers nouveaux et l'information au public de ces dangers; 5) la clause de sauvegarde, pour des actions rapides avec le retrait des ptoduits du marché; 6)l 'imposition des exigences du Protocole de Carthagène sur la biosécurité aux exportateuts communautaires (Advance Informed Agreement Procedure). ${ }^{75}$ Ce n'est peut-être pas un modèle parfait, mais il pourrait inspirer les pays membres du Mercosur.

\footnotetext{
${ }^{74}$ Voir la Commission européenne et la Résolution de Nice.

${ }^{75}$ Voir KNÖR, p.192ss.
} 


\section{II - L'obligation de sécurité en faveur du consommateur et la réparation} des accidents de consommation causés par des OGM: la législation sur la responsabilité civile et le droit international privé du fait de produits avec OGM

Au temps où l'agriculture dominait l'économie, l'obligation de sécurité fut développée par les articles 1245,1945 s. et 1952 s. du Code Civil de 1804 en rapport avec les "choses gardées". "76 Avec l'expansion industrielle et les accidents du travail, la doctrine a d'abord défendu sans succès une interprétation de la notion de faute dans l'article 1382 du Code Civil français comme 'fait générateur de dommages' (Saleilles) ${ }^{77}$ et après -avec succès- a développer sur l'article 1384 du Code Civil française la 'doctrine de la garde de la chose' (Josserand). ${ }^{78} \mathrm{Le}$ gardien de la chose est responsable pour les faits de la chose. La jurisprudence française a développée plus tard l'expression 'obligation de sécuritée. ${ }^{79}$

Aujourd'hui la majorité des législations nationales ont introduit la responsabilité sans faute ou la responsabilité du fait des produits, soit sous la pression de la jurisprudence, soit sous l'influence des organismes internationaux ou d'intégration économique. La question principale en droit de la consommation à notre l'époque post-industrielle conaissant une agriculture génétiquement modifiée, n'est plus de connaître le régime aggravé de la responsabilité civile du fait du produit, mais bien de savoir si les règles existantes sont suffisantes pour developer la prévention des dommages de consommation et des dommages de l'environnement causés par des OGM, et de savoir, en outre, si ces règles sont efficaces en cas d'accident. Le premier défi est la définition du produit lui même, car les OGM peuvent être des organismes vivants ou morts, des organismes pour l'alimentation humaine ou animale, des composants des produits alimentaires ou des produits alimentaires en eux mêmes. Les OGM peuvent être également utisées comme partie d'un service, par exemple un service de restaurant, de 'lunch' etc. Les dommages causés par les OGM ont aussi une spécificité post-industrielle ou post-moderne. La causalité du dommage de l'OGM serait seulement probable et furure, semblable a celle du tabac. Les OGM ont aussi une internationalité émergente, face aux victimes-consommateurs, domiciliées dans plusieurs pays d'exportation de l'OGM, soit vis-à-vis des fournisseurs qui peuvent avoir des plantations, produire des semences, et leurs produits et services en différents pays du monde. Ces dommages peuvent aussi avoir des caractéristiques assez larges ou catastrophiques, signifier même le décès de beaucoup de personnes dans le futur. Voyons d'abord la législation sur la responsabilité civile et après les normes du droit international privé.

\footnotetext{
76 MILLLER, p. 16.

77 Selon MELLIER, p. 17.

78 Selon MELLLER, p. 18.

${ }^{79}$ MELLeR, p. 19 considéré la prenière décision celle de la Cour de Cassation du 21 novembre 1991.
} 


\section{A) La législation sur la responsabilité civile du fait de produits avec OGM}

Aujourd'hui la responsabilité civile du fait des OGM au Brésil est une responsabilité du fait des produits et du fait des services, basée sur la violation du devoir général de sécurité ${ }^{80}$ La loi brésilienne prévoit la responsabilité sans faute dans ces deux cas, aux Art. 12 et 14 de la Loi $8.078 / 90{ }^{81}$ L'idéal serait la présence dans la législation nationale d'une responsabilité civile aggravée et sans faute, spéciale pour les faits des OGM. Les tègles déjà existantes pour les faits des produits, basées sur le risque, peuvent continuer a être utilisées, mais il faudrait à mon avis une norme spéciale pour les OGM, à l'exemple de ceiles relatives aux médicaments.

La base de cette responsabilité spéciale pour les OGM ne doit pas être seulement la 'dangerosité de la chose-OGM', dangerosité qui n'a pas encore été prouvée de façon absolute par la science, ou sa 'garde', comme pour les faits des produits. La responsabilité pour des OGM a une spécificité nouvelle vis-à-vis des produits industriels, car c'est un produit antinaturel agricole qui, au contraire des pesticides agricoles, et semblablement aux remèdes, finit toujours par entrer dans une personne humaine, soit ditectement comme aliment, soit indirectement comme composant, ou aliment des animaux consommés, et d'une façon transformée peut être seulement un composant ou une chose accessoire à un service de consommation. Cette fragmentation et le"double coding" est aussi partie accessoire des services - doivent être relevants pour imposer une responsabilité aggravée et solidaire de tous les fournisseurs concernés.

La base de la responsabilité relative aux OGM et de l'imputation objective de la responsabilité pour le fait d'OGM doit être, à mon avis, la création du produit-OGM ou la décision de création de la chose-OGM (peritus spondet artem suam) et la perturbationrisque volontaire de la nature humaine ou de l'environnement pour des ptofits financiers (cujus commodum ejus periculum).

BD LIMA MARQUES, Contratos, p. 1030s.

${ }^{81}$ Voir mes commentaires, LYMA MARQUES, Contratos, p. $1024 \mathrm{~s}$,

82 JAYME, Erik, Identité culturelle et intégration: Le droit intemationale privé postrmoderne Cours général de droit international privé 1995, in Recueil des Cours de l'Académie de Droit International de la Haye, 1995,II, p. 249. 
Les OGM n'existent pas dans la natute, cela est leur caractéristique principale, à l'instar des ptoduits industriels, des médicaments et des pesticides. Celui qui réalise l'intetvention dans l'organisme, le producteur national ou étranger des semences-OGM par exemple, doit être le premier répondant de cette responsabilité stticte spéciale. La mise en circularion doit être aussi un élément de tesponsabilité objective, l'importateur des grains OGM étant alors aussi solidairment tesponsable des effets néfastes futurs ou ptésents de l'OGM qu'il a inttoduits dans la nature et sur les marchés, en tant que produit agricole ou destiné à l'alimentation animale. La mise en circulation sur. le matché de consommation est le troissième fait imputable, par exemple l'urilisation des composants OGM par les fabricants de soupes et des aliments pout bébés, ou le mélange d'OGM aux hamburgers par un fournisseut de setvices d'une chaîne d'alimentation rapide, ou encore la ente dans les cinémas de pop-cotn de maïs OGM.

La grande question actuelie est celle des commerçants. Le commerçant ou le prestataire de services qui offre des produits OGM, ou avec des composants OGM, doit-il aussi être tesponsable dans cette chaîne de responsabilité? Deux facteurs indiquent qu'il faut inclure les commerçants dans cette règle spéciale de responsabilité aggravée:

1) les produits OGM peuvent être des produits sans indication de l'origine du fabricant, appartenir à la composition d'autres produits, ou faire partie des services d'alimentations (restaurants, hôtels, hospices, cbaînes d'alimentation rapide etc.). Le devoir d'information doit être nécessairement templi par les commerçants ${ }_{2}$ qui ont un contact direct avec le consommateur;

2) si, à l'époque industrielle, les commerçants ont reçu le privilège d'une responsabilité subsidiaire à celle du producteur, comme à l'article 13 de la loi brésilienne, Loi 8.078/90, la décision d'offrir des produits OGM aux consommateur est aujourd'hui une décision qui affecte la qualité-sécurité de sa prestation contractuelle. On peut dire que la décision de ne pas offrir des produits OGM est une décision économique. La mise sur le marché des OGM pour une finalité économique est un tisque accepté par le commerçant (cujus commodum ejus periculum).

Comme on l'a observé, la complexité post-moderne du 'fait dommageable' demande une téglementation spéciale pour la tesponsabilité du fait des OGM. Il faur aussi considérer que les causes d'exclusion de responsabilité applicables aux produits industriels comme pour les risques de développement seraient injustes si elles sont appliquées aux OGM. Aussi la notion de 'défaut' est-elle liée à celle de 'la sécurité à laquelle on peut légitimement s'attendre compte tenu de toutes les circonstances" (Art. 12, $\$ 1$ et Art. 14, $\$ 1$ de la Loi 8.078/90 et aussi Art. 6 de la Directive 85/374/ $\mathrm{CEE}),{ }^{83}$ comme la ptésentation du produit et le moment de la mise en circulation du ptoduit. Alors, si le consommateur est informé par l'étiquetage et les symboles que le produit contient des OGM, et décide malgré cela de le consommer, le défaut de

${ }^{83}$ MARKOVIT\%, p. 372. 
sécurité sera difficilement prouvé, comme on est en train de le voir dans quelques actions contre des fabticants de cigarettes au Brésil.

Aussi, les risques de développement sont des causes d'exonération de responsabilités dans quelques pays. ${ }^{84}$ Le jugement de la Cour de Justice des Communautés européennes rendue le 29 de mai 1997 a montré que les risques de développement sont un facteur objectif d'exonération. La Cour a affirmé que l'article 7 (e) de la Directive 85/374/CEE "ne vise pas spécifiquement la pratique et les normes de sécurité en usage dans le secteur industriel dans lequel opère le producteur, mais, sans aucune restriction, l'état des connaissances scientifiques et techniques, en ce compris son niveau le plus avancé, tel qu'il existait au moment de la mise en circulation du produit en cause.

L'idéal est une règle spéciale pour la responsabilité du fait des OGM, à l'exemple allemand. La règle de responsabilité sans faute pourrait imposer une responsabilité objective sans la nécessité de recourir à l'idée du défaut des produits ou des aliments OGM. Selon l'exemple du paragraphe 32,1 de la loi allemande, Gesetz zur Regelung der Gentechnik/ 1990 , il fallait créer une règle de responsabilité pour dommage pur: "Si quelqu'un souffre d'un dommage à cause des caractéristiques de l'organisme qui a été génétiquement modifié, celui qui a fabriqué, utilisé ou libéré au marché l'OGM doit en répondre." ${ }^{86}$

Si la doctrine préfère continuer a travailler avec l'idée de défaut, il faut bien définir les types de défauts des OGM. À mon avis les défauts de l'OGM peuvent être de deux types: a) défaut de sécurité, défaur de production, manufacture et projection, si l'OGM n'est pas un aliment conforme et sans danger comme l'aliment qu'il templace. Ce défaut là va être imputahle solidairement, soit pour les fabricants de semences er pesticides ("a faillure to design the product so as to avoid danger"), soit pour les producteurs agricoles, soir pour le fournisseurs qui les utilisenr comme ingrédients dans des outres produits ou services ("a failure arising in manufacture so that the product is not as intended" $7,{ }^{87}$ b) défaut d'information, si le producteur de semences, les producteurs agricoles et les fournisseurs de produit et des services dans le marché violent ses devoires d'informer sur la caractéristique OGM des aliments, des produits et parties des services et des ingrédients de produits ("a failure to warm" de caracteristique OGM).

\footnotetext{
${ }^{84}$ Voir TAYLOR, P. 67 ss.

85 Apud TAYLOR, p. 72.

${ }^{36}$ La norme allemande est la suivante: " $\$ 32$ (1) W/rd infolge von Eigenschaften eines Organismus, die auf gentechnischen Arbeiten beruhen, jemand getötet, sein Körper oder seine Gesundheit vexletzt oder eine Sache beschädigt, so ist der Betreiber verpflichtet, den daraus entstehenden Schaden zuersetzen."

${ }^{87}$ Expressions utilisé pour déterminer la nature du déraut pour OWLES, Dertick, The developement of product liability in the USA, Lloyd's Press, London, p. 9.
} 
Une règle de responsabilité sans faute spéciale pour les OGM allait imposé une responsabilité solidaite par loi (voir l'exemple de la loi allemande, $₫ 32,2$ GenTGesetz) et ainsi éviter la discussion sur la nature (contractuelle ou délictuelle) de la responsabilité imposé aux fabticants, producteur industrielle ou agricole, commerçants et fournisseurs des services. La règle pourrait assuré l'indemnisation des dommages morales et matérielles (voir l'exemple de la loi allemande, $\$ 32,4-7$ GenTGesetz) et établie même un limite maximum de responsabilité pour chacun des responsables (voir l'exemple des 85 Millions des EURO de la loi allemande, $₫ 32,7$ GenTGesetz). La prescription et decandence des ces prétentions pourraient suivte les normes générales du fait des produits ou du droit de la consommation, mais le délais doivent commencer à compter seulement après la découverte du dommage et des responsables dans ce cas spéciales (voir l'exemple de loi allemande, $\$ 32,8$ GenTGesetz). La loi peut prévoir un droit d'information spéciale pour les victimes pour découvrir que type de OGM a causé le dommage et qui était le fabricant (Voir l'exemple de la loi allemande, $\$ 35$ GenTGesetz). En suivant le principe du meilleur choix de loi et de régime de responsabilité pour la victime (Günstigkeitsprinzip), ${ }^{88}$ les normes ordinaires sur le fait de produits et la tesponsabilité civile pourrait être utilisé aussi (voir exemple de la loi allemande, $\$ 37$ GenTGesetz). II fallair aussi régler la possibilité de facilitation de la preuve du défaut et du nexus de causalité pour la victime.

Reste encore vérifier le problème de l'internationalité des marchés de consommation d'aujourd'hui.

\section{B) La législation de droit international privé concernant le fait des OGM}

En Droit comparé, on observe que généralement, en cas de fait du produit, tes lois de droit international internationale ptivé nationales indiquent l'application de la loss de l'Etat sur le territoire duquel le fait dommageable s'est produit, la lex loci delicti, comme la loi brésilienne (Art. 9 de la Loi d'introduction au Code Civil, Decreto-Lei 4.657/42) ou indique applicable soit la loi de l'Etat où les effets du dommage se font sentix, comme la ancienne loi allemande et son "Übiquitätstheorie"," ou la loi de l'Etat où le producteur a sont siège,

ab VON HEIN, Jan, Das Günstigkeitsprinzip im Internationalen Deliktrechts, Mohr, Tübingen, 1999, p.2.

8) VON HEIN, p. $89 \mathrm{~s}$. 
comme la loi suisse (Art. 135,1 IPR-Gesetz) ${ }^{90}$ ou le cumule de facteurs, comme celui de la résidence habituelie de la victime et du responsable, comme la nouvelle règle allemande du $\$$ 40,2 EGBGB. ${ }^{\text {II }}$ La jurisprudence aide avec l'application de la loi du 'pays du marché ou le produit été acheté. ${ }^{92}$ Quelques lois prévoissent la possibilité de choix de la loi applicable au delict après le dommage (autonomie de la vonlonté), comme la nouvelle loi allemande, $\$ 42$ EGBGB. Ce choix devait être himite en cas de responsabilité du fait des OGM. Ici, il faut appliquer aussi les règles de droit internationale privé spéciales pour la protection des consommateurs, ${ }^{93}$ qui normalement indiquer un forum privilégie ${ }^{64}$ et des connexions plus flexibles et favorables pour les consommateurs. Les ordres juridiques prévoient des clauses d'exception pour les consommateurs et même l'application de la 'proper law of the tort" ${ }^{95}$ ou la loi avec le lien plus étroite. ${ }^{\%}$

Quelques règles de droit internationale privé sembicnt trop rigide, comme la loi brésilienne qui date de 1942. La tatification de la 'Convention sur la loi applicable à la responsabilité du fait des produits' de la Haye du 2 de octobre $1973^{97}$ pourrait aider le Brésil et d'autres pays a arrivé a la nécessaire flexibilité de connexion en matière d'OGM. La Convention de la Haye de 1973 introduit la notion de responsabilité des fabricants, des producteurs, des fournisseurs de produits et d'auttes personnes, y compris les entrepositaires, constituant la chaine de préparation et distribution commerciale des produits (Art. 3), la convention connait la notion de dommage pour fausse ou manque d'information (Art. 1) et inclut les produits naturels ou agricoles dans les produits qui peuvent causé les dommages (Art. 2). ${ }^{98}$ La Convention prévoit dans les Articles 4,5 et 6 l'application de la loi interne de l'Etat sur le territoire duquel le fait dommageable s'est produit (lex loci delicti) ou la loi de l'Etat de la résidence habituelle de la personne directement lésće. ${ }^{y 9}$ C'est une bonne flexibilité

\footnotetext{
WANDT, p. 72.

"T Voir KADNER GRAZIANO, Thomas, Europäisches Internationales Deliktsrecht, Mohr, Tübingen, 2003, p. 67-68.

92 KADNER GRAZIANO, P. 69.

93 KADNER GRAZIANO, p. 71.

94 Voir WANDT, P. 52s.

"25 Voir la jurisprudence de les Etats Unies, WANDT, p. $142 \mathrm{~s}$.

"Voir KADNER GRAZ,IANO, p. 78.

7 W $A$ NNDT, p. $58 \mathrm{ss}$.

"Voir Conference de La Haye de droit internationale privé, Actes et documents de la douzième session- 2 au 21 octobre 1972, Tome III-Responsabilité du fait des produits, Ed. Bureau Permanent de la Conférence, La Haye, 1974, p. 246-250. Voir aussi WANDT, p. $58 \mathrm{~s}$.

9" Voir 契EITAG, Robert, Der Einfluss des Europäischen Gemischaftsrechts auf das internationale Produkthaftungsrecht, Mohr, Tübinge, 2000, p. $171 \mathrm{ss.}$
} 
de la lex loci delicti commissi, visant appliquer la loi plus favorable à la réparation de la victime, qui est recommandable aussi pour le Brésil. ${ }^{10 \%}$ Les critiques à la Convention de la Haye de 1973 pour l'utilisation de la théorie allemande, Ubiquitätstheorie, d'une façon trop compliqué, ${ }^{101}$ n'est sont pas importanres pour le cas brésilien ${ }^{102}$ La loi brésilienne est trop ancienne et inflexible pour les temps actuels. La ratification de la convention sera déjà un grand progrès. L'autre possibilité est la création d'une tègle spéciale de droit internationale privé pour la responsabilité du fait de l'OGM. La tendance mondiale aujourd'hui est l'utilisation pour des faits des produits et des services de la loi du pays du marché d'acquisition ou utilisation pour les consommateurs. ${ }^{103}$ Alors, on peut suggérer l'application de cette loi d'origine de l'OGM (lex origines ou lois du pays du marché de commercialisation).

\section{Conclusion}

En conclusion, au vue des règles de l'ONU, des règles constitutionnelles et des règles de droit de la consommation brésiliennes, la doctrine pourrait entamer une téflexion sur lidée d'un droit aux informations nécessaites à la santé et dignité humaine, comme par example l'information sur la caractétistique OGM d'un aliment ou les informations sur les médicaments.

L'analyse du cas brésilien, de la législation du Mercosur et de l'Union Européenne indique que le droit de la consommation brésilien a déjà développé des instruments suffisants pour la prévention des dommages ou à tout le moins pour l'information des consommateurs. Le problème est l'efficacité de tels instruments.

\footnotetext{
${ }^{100}$ WANDT, p. 61. Voir sur la flexibilité de la lex loci delicti en droit international privé comparé, LIMA MARQUES, Claudia, Novos xumos do Direito internacional privado quanto às obrigaçōes resultantes de atos ilícitos, in Revista dos Tribunais (São Paulo), vol. 629 (mar. 1988), p. 72-92.

${ }^{111} 1$ KADNER GRAZIANO, p. 77.

102 LIMA MARQUES, Novos rumos, p. 92.

$1{ }^{1} 3$ KADNER GRAZIANO, P. 78.
} 
Comme le commerce internationale actuellement n'est connait presque plus de barrière, ${ }^{304}$ il faut suggérer une action mondial pour assuré l'in formation des consommateurs et les contrôles efficaces, sans préjudice des efforts et sucés du Codex Alimentarius. ${ }^{\text {ins }}$ L'Association Internationale de Droit de la Consommation poutrait artiver à une Résolution avec cette finalité, à l'exemple de las résolutions de l'Association de Droit International, avec des suggestions aux gouvernement des pays. Les suggestions seraient seulement une inspiration pour des futures législations. Mais aujourd'hui, avec la crise de légitimation des institutions gouvernementaux et avec l'esprit post-moderne de narration des objectives, peut-être ces suggestions des professeurs de Droit de la Consommation pourraient tomber dans des champs fertiles.

Au sujet de la prévention des dommages faits aux consommateurs par les OGM au Brésil, il faut suggérer au gouvernement brésilien:

1) la création de mécanismes assurant une évaluation préalable des risques sur la santé publique avant la mise sur le marché des OGM ou des produits contenant des composants OGM ou des produits pour l'alimentation animale; avec OGM.

2) l'imposition de l'étiquetage ou d'une information complète de tous les produits agticoles ou industriels, nationaux ou importés, avec plus de 1\% d'OGM.

3) la création de contrôles administratif́s de surveillance aptès la mise sur le marché des OGM, au moyen de rapports annuels obligatoires aux autorités compétentes provenant des fournisseurs ou importateurs, ou par l'information du public, obligatoire en cas de danger ou de modification de des résultats de la recherche scientifique ou par la surveillance des effets des OGM par les associations de défense du consommateur;

4) la création d'un système transparent d'échange d'informations et d'expériences sur les OGM au sein du Mercosur.

\footnotetext{
${ }^{104} \mathrm{Il}$ faut noter, que les premiers décisions de 'panels' de l'OMC sont sensiblenent contraires aux intérêts de l'environnement, comme dans les cas de la pêthe de 'saumon', la viande aux hormones et les cas de mesures phytosanitaires européennes (voir FABRI, Hélène Ruiz, Le contentieux de l'exécution dans le règlement des différends de l'Organisation mondiale du conmerce, in Joumal du Droit Intermational, n. 3, jul/sept., 2000, p. 605s. et sur les OGM, MALJEAN DUBOIS, p. $975 \mathrm{~s}$.). On parler inclusive d'une "diabolisation de l'OMC", voir MALJIAN-DUBOIS, p. 995. Aussi les premiers décisions arbitrales du Mercosur (http:/ www mregovbr) sont contre les intérêt du consommateur et de la protection de la santé public, même si le Traité d'Asunción créant l'ALADI a dans l'article 50 une clause d'exception au libre commerce pour des motives de santé public et défense du consommateurs, voir détailles dans mon Course à POEA, LIMA MARQUES, p. 657ss. ths Voir NOIVILLE, p. 274s. et MALJEAN-DUBOIS, p. $973 \mathrm{~s}$ et p. 996.
} 
En ce que concerne l'obligation de sécurité et les règles sur la responsabilité, l'Association pourrait suggéter:

1) la création des règles spéciaux pour la responsabilité sans faute et aggravée du fait des OGM. La règle de responsabilité pour du fait OGM ne devait pas permettre l'exclusion de responsabilité pour des risques de développement.

2) la règle de responsabilité sans faute pourtait imposé la responsabilité sans nécessité de recourir à l'idée du défaut des produits ou aliments $O G M$, au modèle de le paragraphe 32,1 de la loi allemande, Gesetz zur Regelung der Gentechnik/1990: "Si quelqu'un soufre un dommage à cause des caractéristiques de l'organisme, qui était génétiquement modifié, celui qui a fabriqué , utilisé ou libéré au marché l'OGM doit répondre."

3) La règle de responsabilité sans faute spéciale pour les OGM devait imposé une responsabilité solidaire par loi aux fabricants, producteur industrielle ou agricole, commerçants et fournisseurs des services.

4) La règle pourrait assuré lindemnisation des dommages morales et matérielles et même un limite maximum de responsabilité pour chacun des responsables.

5) La loi spéciale peut prévoir un droit d'information spéciale pour les victimes pour découvrir que type de OGM ont causé sont dommages et qui était le fabricant. La prescription et décandence des ces prétention pourrait suivre les normes générales du fait des produits ou du droit de la consommation, mais le délais doit commencer à compter seulement après la découverte du dommage et des responsables dans ce cas spéciales.

6) En suivant le principe du meilleur choix de loi et de régime de responsabilité pour la victime, les normes ordinaites sur le fait de produits et la responsabilité civile pourrait être utilisé aussi. Il fallait aussi tégler la possibilité de facilitation de la preuve du défaut et du nexus de causalité pour la victime.

En droit internationale privé, la tendance mondiale actuelle est l'indication pour régler les faits des produits et des services de la loi du pays du marché d'acquisition ou utilisation pour les consommateurs. Alors, on peut suggérer:

1) L'application de la loi d'origine de l'OGM, lex origines ou loi du pays du marché de commercialisation;

2) La tatification de la 'Convention sur la ló applicable à la responsabilité du fait des produits' de la Haye du 2 de octobre de 1973, car la Convention peut aider la réparation des consommateurs en matière du fait internationale d'OGM. 